\title{
Entanglement-Based dc Magnetometry with Separated Ions*
}

\author{
T. Ruster, H. Kaufmann, M. A. Luda, ${ }^{\dagger}$ V. Kaushal, C. T. Schmiegelow, ${ }^{\ddagger}$ F. Schmidt-Kaler, and U. G. Poschinger \\ Institut für Physik, Universität Mainz, Staudingerweg 7, 55128 Mainz, Germany
}

(Received 6 April 2017; revised manuscript received 31 July 2017; published 21 September 2017)

We demonstrate sensing of inhomogeneous dc magnetic fields by employing entangled trapped ions, which are shuttled in a segmented Paul trap. As sensor states, we use Bell states of the type $|\uparrow \downarrow\rangle+\mathrm{e}^{\mathrm{i} \varphi}|\downarrow \uparrow\rangle$ encoded in two ${ }^{40} \mathrm{Ca}^{+}$ions stored at different locations. The linear Zeeman effect leads to the accumulation of a relative phase $\varphi$, which serves for measuring the magnetic-field difference between the constituent locations. Common-mode magnetic-field fluctuations are rejected by the entangled sensor state, which gives rise to excellent sensitivity without employing dynamical decoupling and therefore enables accurate dc sensing. Consecutive measurements on sensor states encoded in the $S_{1 / 2}$ ground state and in the $D_{5 / 2}$ metastable state are used to separate an ac Zeeman shift from the linear dc Zeeman effect. We measure magnetic-field differences over distances of up to $6.2 \mathrm{~mm}$, with accuracies down to $300 \mathrm{fT}$ and sensitivities down to $12 \mathrm{pT} / \sqrt{\mathrm{Hz}}$. Our sensing scheme features spatial resolutions in the 20-nm range. For optimizing the information gain while maintaining a high dynamic range, we implement an algorithm for Bayesian frequency estimation.

DOI: 10.1103/PhysRevX.7.031050

Subject Areas: Atomic and Molecular Physics,

Quantum Information

\section{INTRODUCTION}

Magnetic-field sensors are ubiquitous in modern technology and applied and fundamental research. Various sensing technologies are available, covering different parameter regimes in terms of sensitivity, spatial resolution, bandwidth, and other parameters.

Among the most sensitive magnetic-field sensors are superconducting quantum interference devices (SQUIDs) [1]. These exist in various dimensions, ranging from a few $\mathrm{mm}$ to the tens of $\mathrm{nm}$ regime [2-6]. While SQUIDs are already based on quantum effects, recent advances in quantum technology bring genuine quantum sensors within the reach of applications. Magnetometers based on single well-isolated atomic systems or ensembles have been demonstrated, where the accumulated phase of a

*The U.S. Government is authorized to reproduce and distribute reprints for Governmental purposes notwithstanding any copyright annotation thereon. Any opinions, findings, and conclusions or recommendations expressed in this material are those of the author(s) and do not necessarily reflect the view of the U.S. Army Research Office.

Present address: DEILAP, CITEDEF \& CONICET, J.B. de La Salle 4397, 1603 Villa Martelli, Buenos Aires, Argentina.

*Present address: Departamento de Física, FCEyN, UBA and IFIBA, Conicet, Pabellón 1, Ciudad Universitaria, 1428 Buenos Aires, Argentina.

Published by the American Physical Society under the terms of the Creative Commons Attribution 4.0 International license. Further distribution of this work must maintain attribution to the author(s) and the published article's title, journal citation, and DOI. superposition state during an interrogation time $T$ allows for inference of the ambient magnetic field.

Typically, the choice of a sensing platform requires trading sensitivity versus spatial resolution, as ensemblebased systems are more accurate but also have larger dimensions. Suitable ensemble systems include atomic vapors [7-12], ultracold atomic gases [13,14], and color centers in diamonds [15-17]. Atomic vapors offer record sensitivities below the $1 \mathrm{fT} / \sqrt{\mathrm{Hz}}$ level but have typical dimensions above $1 \mathrm{~mm}^{3}$. In contrast, single vacancy centers [18-21] have been used for high-resolution imaging of magnetic fields in the nm regime. Single trapped ions are also well suited for magnetic-field imaging [22], with spatial resolutions of about $20 \mathrm{~nm}$.

A key parameter for quantum magnetic-field sensing is the interrogation time $T$. For longer interrogation times, more phase can be accumulated during an experimental cycle, such that sensitivity scales as $1 / \sqrt{T}$. In order to prevent dephasing during the course of an experiment, it is crucial to achieve long coherence times by canceling undesired noise sources.

A well-established method for achieving long coherence times is dynamical decoupling [23], where the desired signal is spectrally separated from noise. However, this technique is restricted to measurements of alternating magnetic fields. Recently, dynamical decoupling with a single trapped ion has been used to demonstrate magnetometry in the radio-frequency range, attaining a few-pT $/ \sqrt{\mathrm{Hz}}$ level of sensitivity $[24,25]$. A second option for canceling undesired noise sources is the use of gradiometers, where two identical sensors are used to 
measure magnetic-field gradients while rejecting commonmode fluctuations [26-28].

Quantum entanglement can be harnessed to extend sensing capabilities [29,30]. Entangled GreenbergerHorne-Zeilinger or NOON states can, in principle, yield a sensitivity beyond the standard quantum limit [31-33]. However, an increased sensitivity also implies an increased noise-induced decoherence [34]. Hence, the beneficial effect of entanglement is generally compromised unless measurement schemes are designed to reject noise in favor of the desired signal. With trapped ions, entangled sensor states of the type $\left(|\uparrow \downarrow\rangle+\mathrm{e}^{\mathrm{i} \varphi}|\downarrow \uparrow\rangle\right) / \sqrt{2}$ have been employed to measure local magnetic-field gradients $[35,36]$ as well as the magnetic dipole interaction between the constituents' valence electrons [37].

In this article, we present a magnetic gradiometer, where entangled ions are moved to different locations $x_{1}$ and $x_{2}$ along the trap axis of a segmented linear Paul trap. The dc magnetic-field difference $\Delta B\left(x_{1}, x_{2}\right)$ between the ion locations can be inferred from the phase accumulation rate of these sensor states via the linear Zeeman effect

$$
\Delta \omega\left(x_{1}, x_{2}\right)_{\mathrm{dc}} \equiv \dot{\varphi}_{\mathrm{dc}}=\frac{g \mu_{\mathrm{B}}}{\hbar} \Delta B\left(x_{1}, x_{2}\right) .
$$

Since the net magnetic moment of the two constituent ions vanishes, common-mode noise is rejected such that the coherence time can exceed 20 s [35,36,38,39]. Combined with the fine-positioning capabilities offered by trapped ions, this enables magnetic-field sensing in a parameter regime which could previously not be accessed: We sense dc field differences at around $300 \mathrm{fT}$ precision and $12 \mathrm{pT} / \sqrt{\mathrm{Hz}}$ sensitivity, and the spatial resolution is limited by the size of the ion's ground-state wave function of about $13 \mathrm{~nm}$.

In Sec. II, we describe the procedure for measuring the relative phase $\varphi$ of sensor states, apply it to determine phase accumulation rates $\Delta \omega\left(x_{1}, x_{2}\right)$ in Sec. III, and discuss the limitations in Sec. IV. An efficient measurement scheme utilizing Bayesian frequency estimation is presented in Sec. V. In Sec. VI, we extend our sensing scheme to infer both dc and ac magnetic-field differences from the measured phase accumulation rates. Finally, in Sec. VII, we compare our results to state-of-the-art magnetic-field measurement techniques and discuss applications of our sensor.

\section{EXPERIMENTAL PROCEDURE}

We trap two ${ }^{40} \mathrm{Ca}^{+}$ions in a segmented linear Paul trap [40], featuring 32 control electrode pairs along the trap axis $x$. The distance between the center of neighboring electrodes is $200 \mu \mathrm{m}$. A dc trapping voltage of $-6 \mathrm{~V}$ leads to an oscillation frequency of the ions of about $1.5 \mathrm{MHz}$ along the trap axis, corresponding to a $1 \sigma$ width of the ground-state wave function of about $13 \mathrm{~nm}$.

A quantizing magnetic field at an angle of $45^{\circ}$ to the trap axis is created by $\mathrm{Sm}_{2} \mathrm{Co}_{17}$ permanent magnets, splitting the ground-state Zeeman sublevels $|\downarrow\rangle \equiv\left|S_{1 / 2}, m_{j}=-\frac{1}{2}\right\rangle$ and $|\uparrow\rangle \equiv\left|S_{1 / 2}, m_{j}=+\frac{1}{2}\right\rangle$ by about $2 \pi \times 10.4 \mathrm{MHz}$. The trap setup is shielded from ambient magnetic-field fluctuations by a $\mu$-metal magnetic shielding enclosure, yielding a coherence time of about $300 \mathrm{~ms}$ [41] in a Ramsey-type experiment.

Laser cooling, coherent spin manipulations, and read-out [42] take place in the laser interaction zone (LIZ) of the trap (Fig. 1). An experimental cycle starts with Doppler laser cooling a two-ion crystal on the $S_{1 / 2} \leftrightarrow P_{1 / 2}$ cycling transition near $397 \mathrm{~nm}$. All collective transverse modes of vibration of the ion crystal are cooled close to the motional ground state via resolved sideband cooling on the stimulated Raman transition between $|\uparrow\rangle$ and $|\downarrow\rangle$. State initialization to $|\uparrow \uparrow\rangle$ is achieved via frequency-selective pumping utilizing the narrow $S_{1 / 2} \leftrightarrow D_{5 / 2}$ quadrupole transition near $729 \mathrm{~nm}$.

A pair of copropagating laser beams, detuned by $2 \pi \times$ $300 \mathrm{GHz}$ from the cycling transition, serves to drive spin rotations without coupling to motional degrees of freedom. After state initialization, a $\pi / 2$ pulse on both ions creates the superposition state $|\uparrow \uparrow\rangle+\mathrm{i}|\uparrow \downarrow\rangle+\mathrm{i}|\downarrow \uparrow\rangle-|\downarrow \downarrow\rangle$. Then, an entangling geometric phase gate [43] is carried out. A spin-dependent optical dipole force transiently excites collective vibrations only for parallel spin configurations, such that the $|\uparrow \uparrow\rangle$ and $|\downarrow \downarrow\rangle$ states acquire a phase of $\pi / 2$.

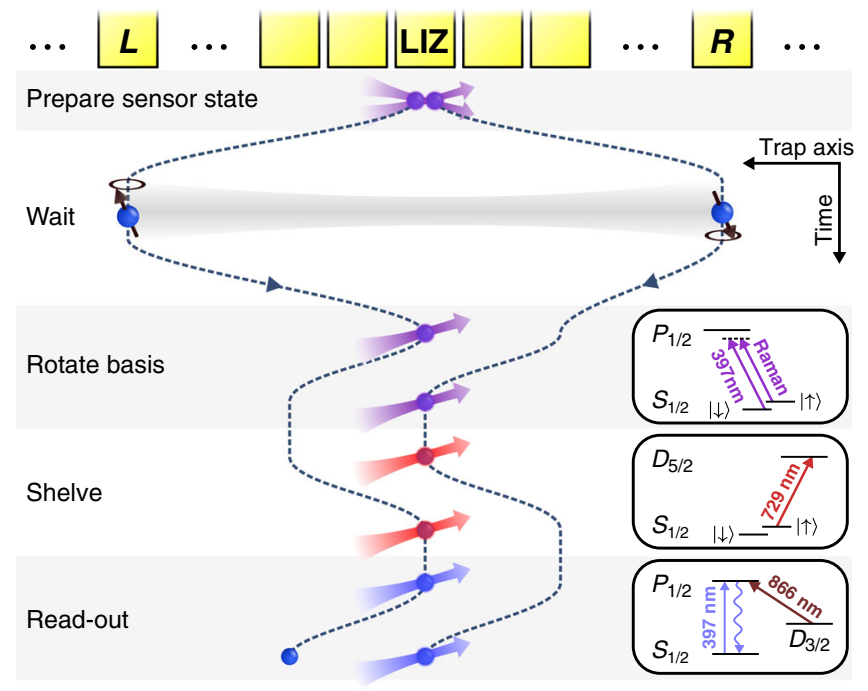

FIG. 1. Experimental procedure for measurements of inhomogeneous magnetic fields. After creation of the sensor state at the LIZ, the two constituent ions are separated and shuttled to the desired trap segments $L$ and $R$. In order to measure the accumulated phase during the interrogation time $T$, the ions are individually shuttled to the LIZ to perform basis rotations that allow for state read-out via electron shelving and fluorescence detection in either the $\hat{X}_{1} \hat{X}_{2}$ or $\hat{X}_{1} \hat{Y}_{2}$ basis. For basis rotations, electron shelving, and fluorescence detection, the relevant energy levels are shown. 
The optical dipole force is generated by two orthogonally propagating laser beams that provide spin-motion coupling only to the transverse vibrational modes of the ion crystal. After the phase gate, two additional $\pi / 2$ pulses are applied to both ions, with phase shifts of $0^{\circ}$ and $-45^{\circ}$ with respect to the initial $\pi / 2$ pulse. These pulses lead to the sensor state $(|\uparrow \downarrow\rangle+|\downarrow \uparrow\rangle) / \sqrt{2}$, and a state fidelity of 99.2 (5)\% is attained (see Ref. [44]).

For the subsequent operations, the two-ion crystal is separated [45,46]. Ion movement along the trap axis is controlled by applying time-dependent voltages on individual trap electrodes via a fast multichannel arbitrary waveform generator at update rates of up to $2.5 \mathrm{MSamples} / \mathrm{s}$ [47]. After separation, the ions are shuttled to the desired locations $x_{1}$ and $x_{2}$ with a maximum distance of $6.2 \mathrm{~mm}$. The ions are kept at these locations for an interrogation time $T$. Any inhomogeneity of the magnetic field leads to the accumulation of a phase $\varphi\left(x_{1}, x_{2}, T\right)$ according to Eq. (1), resulting in the state $\left(|\uparrow \downarrow\rangle+\mathrm{e}^{\mathrm{i} \varphi}|\downarrow \uparrow\rangle\right) / \sqrt{2}$.

After the interrogation time $T$, both ions are consecutively moved back to the laser interaction zone for spin read-out. There, local spin rotations are driven in order to measure the spin along a given basis. Then, population in the state $|\uparrow\rangle$ is selectively transferred for each ion via laserdriven rapid adiabatic passage to the metastable $D_{5 / 2}$ state, followed by conditional detection of resonance fluorescence via a photomultiplier tube while driving the cycling transition (see Fig. 1, inset).

Rather than fully reconstructing the quantum state by measuring in nine different bases, we reduce the number of required measurements by parametrizing the density matrix describing the spin state of the two ions as

$$
\hat{\rho}=\frac{1}{2}\left(\begin{array}{cccc}
0 & 0 & 0 & 0 \\
0 & 1 & C e^{-\mathrm{i} \varphi} & 0 \\
0 & C e^{\mathrm{i} \varphi} & 1 & 0 \\
0 & 0 & 0 & 0
\end{array}\right)
$$

in the logical basis $\{|\uparrow \uparrow\rangle,|\uparrow \downarrow\rangle,|\downarrow \uparrow\rangle,|\downarrow \downarrow\rangle\}$, with the parity contrast $0 \leq C \leq 1$. This form closely resembles the measured density matrix of the sensor state. As shown in Appendix A, for inference of the phase $\varphi$ and contrast $C$, it is sufficient to measure the parity of the two operators $\hat{X}_{1} \hat{X}_{2}$ and $\hat{X}_{1} \hat{Y}_{2}$. Thus, upon measuring the operators $\left\{\hat{X}_{1} \hat{X}_{2}, \hat{X}_{1} \hat{Y}_{2}\right\}$ each $\{N, M\}$ times, $\varphi$ and $C$ are determined from the number of events $\{n, m\}$ where the state has been projected to either $|\uparrow \uparrow\rangle$ or $|\downarrow \downarrow\rangle$. The phase $\varphi$ and contrast $C$ are extracted from the parity results via maximum likelihood estimation. The employed phase estimation method is robust against deviations of the state from the form of Eq. (2) [44].

\section{PHASE ACCUMULATION MEASUREMENTS}

In order to determine the phase accumulation rate $\Delta \omega\left(x_{1}, x_{2}\right)$ of the sensor state with both high sensitivity and high dynamic range, a measurement scheme is required that takes the $2 \pi$ ambiguity of phase measurements into account. Recently, measurement schemes [48-50] have been demonstrated, where phase measurements at a few fixed interrogation times are used to resolve this ambiguity. In these schemes, interrogation times and weight factors are carefully chosen to find an optimal balance between sensitivity and dynamic range. Better performance for high-dynamic range sensors is potentially offered by measurement schemes that adapt the interrogation time based on prior knowledge [51]. Since these typically require a more complex implementation, the choice of a measurement scheme depends on the desired dynamic range of the sensor.

In a straightforward incremental approach, we consecutively perform phase measurements at slowly increasing, predefined interrogation times in order to estimate the required dynamic range of our sensor. A linear fit to the function

$$
\varphi\left(T ; x_{1}, x_{2}\right)=\varphi_{0}\left(x_{1}, x_{2}\right)+\Delta \omega\left(x_{1}, x_{2}\right) \times T
$$

reveals the phase accumulation rate $\Delta \omega\left(x_{1}, x_{2}\right)$ and a phase offset $\varphi_{0}\left(x_{1}, x_{2}\right)$. The constant phase offset is accumulated during the ion movement in the inhomogeneous magnetic field. For each phase measurement at interrogation time $T$, the resulting phase $\varphi_{\text {meas }}\left(T ; x_{1}, x_{2}\right)$ is incremented or decremented by multiples of $2 \pi$ until it falls within a range of $\pm \pi$ to the fit function resulting from previous phase

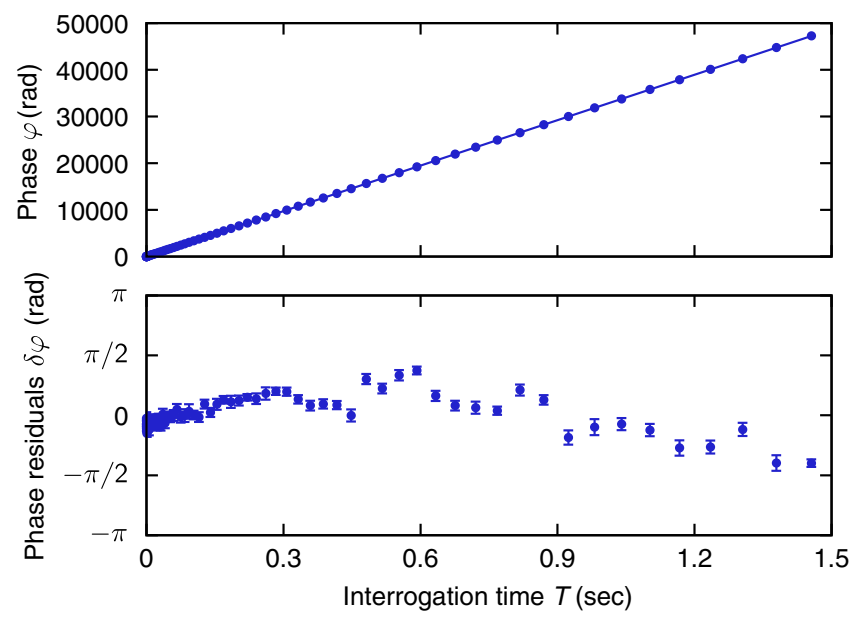

FIG. 2. Incremental measurement of the phase accumulation rate $\Delta \omega$ at an ion distance of $d=6.2 \mathrm{~mm}$. A linear fit to measurements of the accumulated phase $\varphi$ at predefined interrogation times (top part), and the fit residuals $\delta \varphi$ for each phase measurement are shown (bottom part). For each point, measurements of both operators have been repeated 50 times. 
measurements, i.e., $\left|\varphi\left(T ; x_{1}, x_{2}\right)-\varphi_{\text {meas }}\left(T ; x_{1}, x_{2}\right)\right|<\pi$. In order to check if the phase has been incremented or decremented properly, we verify that the residuals of all points are well below $\pi$. Figure 2 shows an example measurement at maximum ion distance $d=6.2 \mathrm{~mm}$ and the residuals $\delta \varphi$ for each point. In this measurement, phases of over 40000 rad have been accumulated during interrogation times of up to $T_{\max }=1.5 \mathrm{~s}$, but the residuals $|\delta \varphi|$ of all measurement points are well below $\pi$.

The maximum interrogation time $T_{\max }$ is ultimately limited by the coherence time $T_{\text {coh }}$ of the sensor state. The coherence time is therefore analyzed in the following section.

\section{COHERENCE TIMES}

We characterize the coherence time $T_{\text {coh }}$ of the sensor state for two settings: The ions are kept (i) in a common potential well at a distance of about $4.2 \mu \mathrm{m}$ and (ii) in separate harmonic wells at the maximum possible distance of $6.2 \mathrm{~mm}$. The coherence time is inferred from measurements of the contrast $C$ for varying interrogation times $T$. For each interrogation time, we repeat the experimental
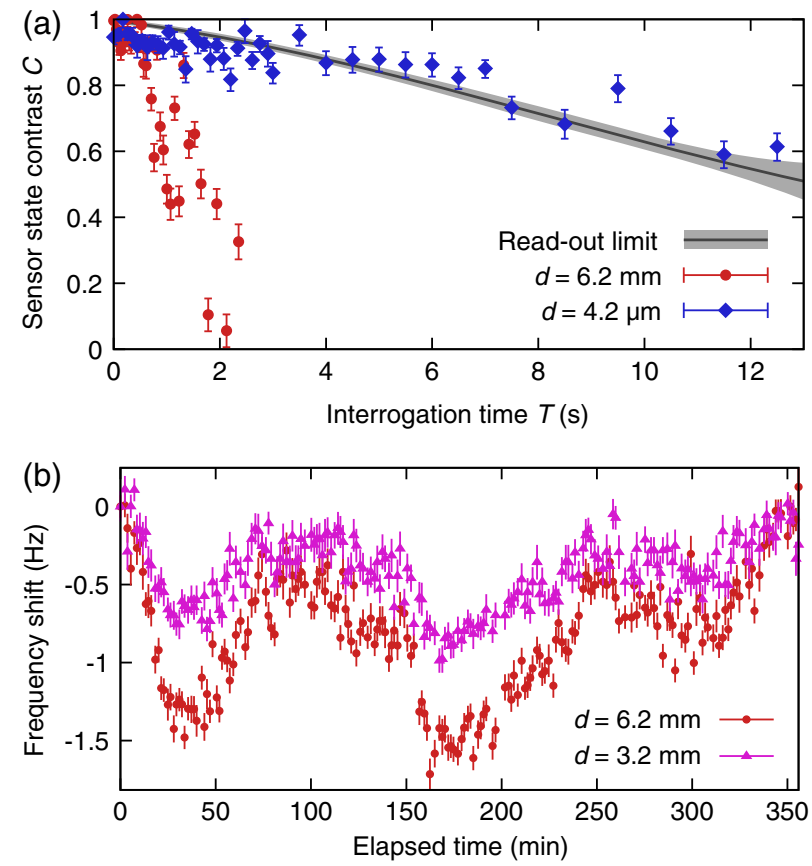

FIG. 3. (a) Sensor state contrast $C$ versus interrogation time $T$ at the maximum ion distance of $d=6.2 \mathrm{~mm}$ (red dots) and at an ion distance of $d=4.2 \mu \mathrm{m}$ (blue squares). For illustration, the black curve and gray region indicate a third-order polynomial fit to a separate read-out fidelity measurement and its confidence bands. (b) Simultaneous drift of the measured frequency difference for ion distances $d=6.2 \mathrm{~mm}$ (blue circles) and $d=3.2 \mathrm{~mm}$ (purple triangles) over a duration of about 6 hours with an interrogation time of $T=150 \mathrm{~ms}$. For $d=3.2 \mathrm{~mm}$, the measured drift is suppressed by a factor of about 1.6 as compared to the maximum ion distance. procedure 400 times for each of the two measurement operators.

For case (i), a coherence time $T_{\text {coh }}>12.5 \mathrm{~s}$ is observed [Fig. 3(a)]. In this regime, residual heating of the radial modes of motion compromises the fidelity of electron shelving and therefore the spin read-out. In separate measurements, we characterized the spin read-out efficiency for the input states $|\uparrow \uparrow\rangle$ and $|\downarrow \downarrow\rangle$, and confirmed that the observed contrast loss is entirely caused by read-out.

For the maximum possible ion distance, a Gaussian contrast decay is observed, with a coherence time in the 1-2-s range. For Gaussian contrast decay, the best sensitivity for our phase measurement scheme is achieved at an interrogation time corresponding to a contrast of 0.85 (see Ref. [44]).

The contrast decay at maximum ion distance is presumably caused by a slow drift of the magnetic-field minimum position along the trap axis. In order to verify our presumption, we measured this drift consecutively for two different ion separation distances of $d=6.2 \mathrm{~mm}$ and $d=3.2 \mathrm{~mm}$ over the course of 6 hours [Fig. 3(b)]. For the former case, a typical drift rate of $1 \mathrm{~Hz} / \mathrm{h}$ is observed. We verified that this drift rate corresponds to a contrast decay within $2 \mathrm{~s}$. For an ion distance of $d=3.2 \mathrm{~mm}$, the drift rate is suppressed by a factor of about 1.6 as compared to the maximum ion distance. The spatial dependence of the observed drift rates is consistent with movement of the ion trap relative to the magnetic field in the 200-nm range, equivalent to thermal expansion of our vacuum chamber due to a temperature change of roughly $0.1^{\circ} \mathrm{C}$.

\section{BAYESIAN FREQUENCY ESTIMATION}

In order to speed up the incremental measurement scheme for determining $\Delta \omega\left(x_{1}, x_{2}\right)$ described in Sec. III, we implement an adaptive scheme for frequency estimation based on a Bayesian experiment design algorithm [51,52].

In general, such algorithms control the choice of a measurement parameter-in our particular case, the interrogation time - which, in each measurement run, guarantees the optimum gain of information on the parameter to be determined. These algorithms are beneficial in situations where only a few parameters are to be determined, an accurate model relating the design parameters to the measurement outcome holds, and the measurement is "expensive" in terms of resources such as time.

In Bayesian statistics, for a given phase measurement to be carried out, the combined result of all previous measurements is expressed with the prior probability distribution function (PDF) $p\left(\Delta \omega, \varphi_{0}\right)$. Initially, we assume a uniformly distributed prior PDF, limited to a reasonable parameter range $\Delta \omega \in\left\{\Delta \omega_{\min }, \Delta \omega_{\max }\right\}$ and $\varphi_{0} \in\{-\pi, \pi\}$. After a phase measurement with the outcome $\{n, m\}$, the combined result is described by the posterior PDF, 
$\tilde{p}\left(\Delta \omega, \varphi_{0} \mid n, m ; T\right)=\frac{p\left(n, m \mid \Delta \omega, \varphi_{0} ; T\right) p\left(\Delta \omega, \varphi_{0}\right)}{p(n, m \mid T)}$

with the update function $p\left(n, m \mid \Delta \omega, \varphi_{0} ; T\right)$, as given by the likelihood function (see Appendix B). The marginal PDF $p(n, m \mid T)$ ensures normalization of the posterior. Hence, the posterior can just be normalized, and computing the marginal is not required.

The interrogation time $T$ for each phase measurement is calculated such that the expected increase of the Shannon information in the posterior PDF is maximized (see Appendix C). With this approach, we observe that the automated measurement operates in two distinct measurement regimes: The measurement starts in the capture regime, where $T$ is consecutively increased from $T=0$ to the desired maximum time $T_{\max }$ in order to unambiguously identify $\Delta \omega$ without any previous information on its value. Then, in the tracking regime, the algorithm alternates $T$ between $T_{\max }$ and $T=0$ for the best sensitivity. In order to efficiently track drifts of $\Delta \omega$, we intentionally cause a "memory loss" by broadening the prior PDF by about 5\% of its width for tracking phase measurements at $T_{\max }$. This facilitates the determination of frequencies that deviate from the previous mean value.

Figure 4 visualizes an example measurement. In Fig. 4(a), the update functions of the first phase measurements in the capture regime are shown. It can be seen that a single phase measurement alone is not sufficient to estimate $\Delta \omega$. However, the combined result of multiple phase measurements yields an approximate Gaussian marginal distribution of $\Delta \omega$, from which the mean value $\langle\Delta \omega\rangle$ and the standard error $\Delta \omega_{\text {err }}$ are inferred. Figure 4(b) depicts the interrogation time $T$ for each experimental cycle and the standard errors of the results, versus the total elapsed time of the measurement. In the capture regime, the measurement error scales as $\Delta \omega_{\text {err }} \propto 1 / t^{1.8(2)}$, with the elapsed measurement time $t$. This is a significant improvement over recent high-dynamic-range measurement schemes with fixed interrogation times, in which error scalings close to $1 / t$ have been demonstrated [48-50]. The maximum interrogation time is reached after about $t=12 \mathrm{~min}$, which is about 10 times faster than in the incremental measurement scheme.

In the tracking regime, the error scaling is reduced to $\Delta \omega_{\text {err }} \propto 1 / \sqrt{t}$, and the precision limit given by the magnetic-field inhomogeneity drift rates and the coherence time is approached. A minimum error of $\Delta \omega_{\text {err }}=$ $2 \pi \times 2.5 \mathrm{mHz}$ is obtained. Now, the uncertainty after each measurement is no longer reduced, but the parameter estimates are corrected for drifts [see Fig. 4(c)].

The shot-noise-limited sensitivity describes the minimal frequency change that can be discriminated within unit time:

$$
S_{\omega}=\Delta \omega_{\text {err }} \sqrt{T_{\text {tot }}}
$$

with the standard error of the frequency measurement $\Delta \omega_{\mathrm{err}}$, which has been achieved during a total experimental time of $T_{\text {tot }}$ [53]. As the sensitivity depends on the chosen interrogation time, we calculate $S_{\omega}$ separately for each phase measurement, only taking prior knowledge of the phase offset $\varphi_{0}$ into account. At an ion distance of $d=800 \mu \mathrm{m}$, a best sensitivity of $S_{\omega}=2 \pi \times 116 \mathrm{mHz} / \sqrt{\mathrm{Hz}}$ is obtained for an interrogation time of $T_{\max }=3.0 \mathrm{~s}$. At this interrogation time, we obtained a mean contrast $C$ of about 0.94 and an average duration of $3.3 \mathrm{~s}$ for a single experimental cycle; i.e., about $91 \%$ of the measurement time has been utilized for phase accumulation. Thus, we reach $79 \%$ of the theoretical standard quantum limit of $1 / \sqrt{T_{\max }}=2 \pi \times 92 \mathrm{mHz} / \sqrt{\mathrm{Hz}}$. Our results are on par with recent measurements of ac


FIG. 4. Bayesian evaluation of a measurement at an ion distance of $d=800 \mu \mathrm{m}$. (a) Update functions (colored bars) in $\left(\Delta \omega, \varphi_{0}\right)$ parameter space of the first four phase measurements with $N=M=50$ repetitions of the experimental procedure and the posterior PDF (purple ellipse) after these iterations. (b) Interrogation times (blue bars) and error of the frequency determination (green points) for each phase measurement versus elapsed measurement time. (c) The posterior PDFs at maximum interrogation time for subsequent phase measurements are visualized by open ellipses, corresponding to the $39.4 \%$-credible regions. One can see that in this regime, the measurement is tracking the drift of the magnetic-field difference (see text). The posterior PDF pertaining to the last measurements is indicated as a density plot. 


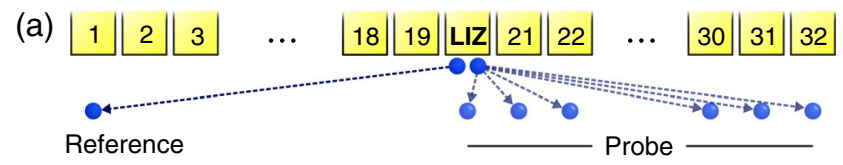

(b)



(c)

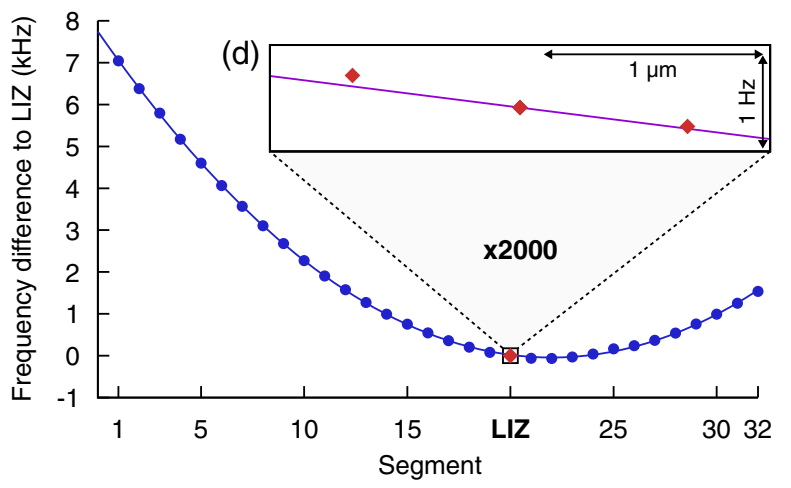

FIG. 5. Frequency differences from the LIZ. A sensor state is prepared, and the probe ion is moved to an arbitrary desired position. At the same time, the reference ion is either moved to (a) segment 1 or (b) segment 32 to maximize the ion distance. The results for each segment are shown in panel (c). In diagram (d), high-precision measurements close to the LIZ yield standard errors for the ion position and frequency difference of about $10 \mathrm{~nm}$ and $2 \pi \times 10 \mathrm{mHz}$, respectively.

magnetic fields with single ions [24], only surpassed by sensors with larger dimensions $[8,16]$.

We utilize our measurement scheme for mapping the frequency difference $\Delta \omega$ to the laser interaction zone (segment 20) along the trap axis. We perform a frequency measurement for each trap segment, where we move one ion, the probe ion, to the desired segment and the second reference ion to either segment 1 , if the probe ion is being moved to segments $20-32$, or to segment 32 , if the probe ion is being moved to segments 1-20 [Figs. 5(a) and 5(b)]. This way, the ion distance is sufficiently large such that the trapping potential of the reference ion does not affect the probe ion position at the given level of accuracy and vice versa. The results are depicted in Fig. 5(c). In Fig. 5(d), additional measurements close to the laser interaction zone are shown, allowing us to infer frequency gradients with high precision. For reaching spatial accuracies of about $10 \mathrm{~nm}$, the probe ion position has been calibrated via an EMCCD camera (see Ref. [44]).

\section{SEPARATION OF DC AND AC ZEEMAN SHIFTS}

In addition to the static Zeeman effect [Eq. (1)], the energy levels of the sensor state are also shifted by the ac
Zeeman effect due to oscillating magnetic fields. This shift is caused by off-resonant driving of the magnetic dipole transition between neighboring magnetic sublevels of a given electronic state [54]. In our experimental setting, such oscillating magnetic fields are generated by the charging and discharging currents of the radio-frequency (rf) electrodes of the Paul trap. In an ideal symmetric trap, the equilibrium positions of the ions are located on the nodal line of the rf field, where the magnetic fields also cancel out. However, residual displacement from the rf node due to stray electric fields and trap fabrication imperfections gives rise to a position-dependent frequency shift between the populated magnetic sublevels [44]:

$$
\omega^{(\mathrm{ac})}(x)=\Delta m_{j}\left(g \frac{\mu_{\mathrm{B}}}{2 \hbar} B_{\mathrm{rf}, \perp}(x)\right)^{2} \frac{\nu(x)}{\nu(x)^{2}-\Omega_{\mathrm{rf}}^{2}} .
$$

Here, $x$ is the ion position along the trap axis, $B_{\mathrm{rf}, \perp}(x)$ is the component of the oscillating magnetic field perpendicular to the static quantizing magnetic field, $\Omega_{\mathrm{rf}}=2 \pi \times 33 \mathrm{MHz}$ is the trap drive frequency, and $\nu(x)$ denotes the total (angular) frequency splitting between neighboring $\left(\left|\Delta m_{j}\right|=1\right)$ Zeeman sublevels.

For sensor states encoded in different electronic state manifolds, the respective Landé factors lead to different contributions to the total phase accumulation rates from dc and ac fields. Hence, by encoding entangled sensor states within different electronic states of ${ }^{40} \mathrm{Ca}^{+}$, our sensing scheme is extended to distinguish between ac and dc magnetic fields. We utilize the $m_{j}= \pm 5 / 2$ sublevels of the metastable $D_{5 / 2}$ state in addition to the $S_{1 / 2}$ ground state for frequency-difference measurements. We prepare the sensor state $|+5 / 2,-5 / 2\rangle+|-5 / 2,+5 / 2\rangle$ by first preparing the state $|\uparrow \downarrow\rangle+|\downarrow \uparrow\rangle$ and then transferring the populations of both ions to the respective sublevels of the $D_{5 / 2}$ metastable state, i.e., $|\uparrow\rangle \rightarrow|+5 / 2\rangle$ and $|\downarrow\rangle \rightarrow$ $|-5 / 2\rangle$ [Fig. 6(a)]. The population transfer is carried out via composite inversion laser pulses near $729 \mathrm{~nm}$ [55]. Considering the Landé factors of both states $g_{S}=$ 2.00225664(9) [56] and $g_{D}=1.2003340(3)$ [57], the
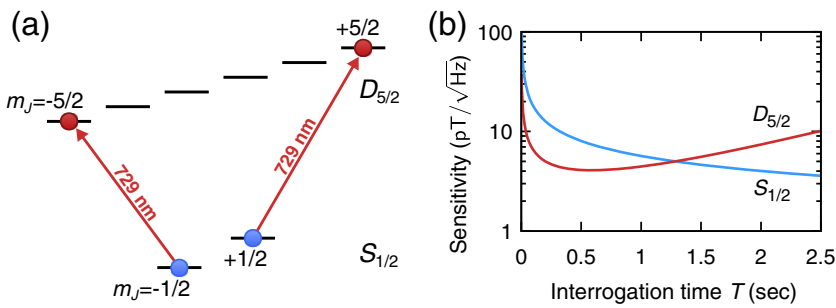

FIG. 6. (a) Relevant transitions for creating the $D_{5 / 2}$ sensor state. (b) Theoretical shot-noise limited sensitivity of magneticfield measurements versus interrogation time $T$ for both $S_{1 / 2}$ and $D_{5 / 2}$ sensor states, showing how spontaneous decay limits the sensitivity of the $D_{5 / 2}$ sensor state for long interrogation times (see text). 
$D_{5 / 2}$ sensor state features phase accumulation rates that are increased by a factor of 3 . However, spontaneous decay at a rate of $1 / \tau$ per ion leads to a reduction of valid measurement cycles, with $\tau=1.168(7) \mathrm{s}$ [58]. We employ an additional fluorescence detection step before state read-out to reject measurements where at least one ion has decayed from the $D_{5 / 2}$ state. Beyond wait times of $\tau / 2$, this postselection reduces the sensitivity of the measurement [Fig. 6(b)].

Measurement of both differential phase accumulation rates $\Delta \omega_{S}$ and $\Delta \omega_{D}$ [59] for the respective $S_{1 / 2}$ and $D_{5 / 2}$ sensor states allows for the disambiguation of the static magnetic-field difference and the differential ac Zeeman shift. As the sensor states are affected differently by the static dc Zeeman effect and the ac Zeeman shift, we can infer the magnetic-field difference via (see Ref. [44])

$$
\Delta B=\frac{\hbar}{\mu_{B}} \frac{\Delta \omega_{D}-\chi \Delta \omega_{S}}{5 g_{D}-\chi g_{S}} .
$$

Here, $\chi=\Delta \omega_{D}^{(\mathrm{ac})} / \Delta \omega_{S}^{(\mathrm{ac})}$ denotes the ratio of the differential ac Zeeman shifts pertaining to the $D_{5 / 2}$ and $S_{1 / 2}$ sensor states. The differential ac Zeeman shift between the constituent ions of the $S_{1 / 2}$ sensor state is given by

$$
\Delta \omega_{S}^{(\mathrm{ac})}=\Delta \omega_{S}-g_{S} \frac{\mu_{B}}{\hbar} \Delta B .
$$

Under the approximation that the magnetic-field inhomogeneity is small compared to the absolute magnetic field, i.e., the energy splittings $\nu_{S}(x)$ and $\nu_{D}(x)$ of the respective electronic states are constant along the trap axis, $\chi$ is calculated via

$$
\chi \approx 5\left(\frac{g_{D}}{g_{S}}\right)^{2} \frac{\nu_{D}}{\nu_{S}} \times \frac{\nu_{S}^{2}-\Omega_{\mathrm{rf}}^{2}}{\nu_{D}^{2}-\Omega_{\mathrm{rf}}^{2}} .
$$

This approximation is well fulfilled in our experimental setup.

Experimentally, we measure the phase accumulation rates $\Delta \omega_{S}$ and $\Delta \omega_{D}$ by performing alternating experimental cycles on the $S_{1 / 2}$ and $D_{5 / 2}$ sensor states. The respective interrogation times $T_{S}$ and $T_{D}$ are individually determined by the Bayesian algorithm. Additional measurements on a single ion at the laser interaction zone are employed to determine the transition frequencies $\nu_{S}\left(x_{\mathrm{LIZ}}\right), \nu_{D}\left(x_{\mathrm{LIZ}}\right)$, and the absolute ac Zeeman shift $\omega_{S}^{(\mathrm{ac})}\left(x_{\text {LIZ }}\right)$ (see Ref. [44]). The transition frequencies $\nu_{S}\left(x_{\mathrm{LIZ}}\right)$ and $\nu_{D}\left(x_{\mathrm{LIZ}}\right)$ are plugged into the ac Zeeman ratio $\chi$ [Eq. (9)], which is used to infer $\Delta B$ [Eq. (7)] and $\Delta \omega_{S}^{(\text {ac) }}$ [Eq. (8)].

Figure 7 depicts the absolute ac Zeeman shift along the trap axis. At the laser interaction zone, an ac Zeeman shift of $\omega_{S}^{(\mathrm{ac})}\left(x_{\mathrm{LIZ}}\right)=-2 \pi \times 0.93(12) \mathrm{Hz}$ is revealed. For remote segments, the magnitude of the frequency shift

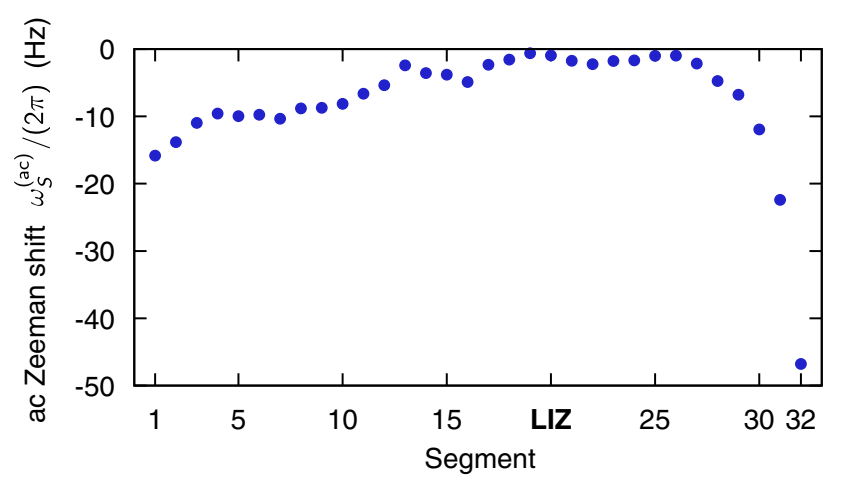

FIG. 7. Frequency shift of the $S_{1 / 2}$ sensor state due to the ac Zeeman effect along the trap axis. Within the error bars of about $2 \pi \times 0.2 \mathrm{~Hz}$, the ac Zeeman shift is similar for both $S_{1 / 2}$ and $D_{5 / 2}$ sensor states.

increases by up to $2 \pi \times 50 \mathrm{~Hz}$. This behavior is presumably caused by a displacement of the ions' equilibrium positions from the nodal line of the rf field, which are minimized only at the laser interaction zone to compensate excess micromotion. For all ion positions, standard errors of about $\omega_{S \text {,err }}^{(\mathrm{ac})}=2 \pi \times 0.2 \mathrm{~Hz}$ are reached. Compared to recent measurements of the ac Zeeman shift arising from microwave fields [22], this is an improvement by 3 orders of magnitude. Thus, our measurement technique may be used to improve the fidelity of microwave-driven quantum gates, where precise mapping of the ac Zeeman shift is important.

For dc magnetic-field differences, we attain sensitivities down to $S_{B}=12 \mathrm{pT} / \sqrt{\mathrm{Hz}}$ at interrogation times of $T_{S}=$ $1.50 \mathrm{~s}$ and $T_{D}=0.48 \mathrm{~s}$. Precision as good as $\Delta B_{\text {err }}^{\text {(stat) }}=$ $310 \mathrm{fT}$ is reached at an ion distance of $d=800 \mu \mathrm{m}$.

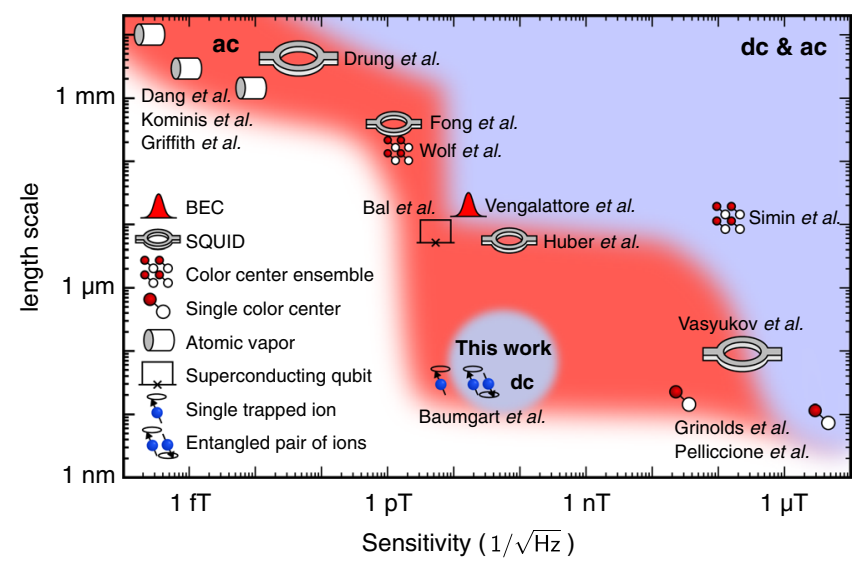

FIG. 8. Overview of recently developed magnetic-field sensors in a size-sensitivity diagram $[2,3,5,6,9-11,14-16,20,21,24,60]$ : The shaded area represents the accessible regime for $\mathrm{dc}$ and quasistatic $<1 \mathrm{~Hz}$ (blue) or ac (red) fields. The regime for dc sensing is substantially extended by this work. 


\section{CONCLUSION AND OUTLOOK}

We have demonstrated a novel magnetometry scheme harnessing entangled ions, which are freely positioned in a segmented Paul trap. The long coherence times of the entangled states enable precise measurement of dc magnetic-field differences. Combined with the high spatial resolution offered by trapped ions, the accessed parameter regime is unique so far among magnetic-field sensors (Fig. 8).

Our measurement scheme additionally characterizes the position-dependent ac Zeeman effect due to the rf trap drive in Paul traps, which is a hard-to-characterize source of errors for precision measurements in frequency standards. For recent optical clocks, the ac Zeeman shift contributes to the fractional error in the $10^{-20}-10^{-17}$ range [61-63].

Precise knowledge of the magnetic field along the trap axis is essential for a shuttling-based approach towards scalable quantum-information experiments in Paul traps. In this approach, quantum algorithms are carried out with multiple ions residing at different trap segments, where different phases are accumulated. These phases have to be taken into account within the computational sequences [64].

The benefits of the presented measurement technique could be harnessed to characterize the magnetic properties of samples, which are small compared to the size of the ion trap. In this case, the absolute magnetic field of the sample is accessible if the reference ion is placed sufficiently far away. The presented gradiometer could be extended to a full-fledged dc vector magnetometer: By changing the direction of the quantizing magnetic field, the absolute magnetic field of the sample along all directions can be measured. Suitable samples to be probed include additional trapped ions, neutral atoms trapped by optical dipole forces [65], or more complex samples such as single-molecule magnets [66]. In the latter case of single neutral atoms or cold quantum gases, the absence of coulomb repulsion allows the probe ion to be placed in a $\mathrm{nm}$ distance to or within the sample.

For sensing of sample surfaces, the achievable accuracy of our method is limited by increased heating via fluctuating electric fields in proximity to the surface, which can deteriorate the read-out fidelity. This effect, however, strongly depends on the characteristics of the sample, such as temperature, surface structure, and contamination, where the underlying mechanisms are not yet entirely understood [67].

\section{ACKNOWLEDGMENTS}

We acknowledge former contributions from Claudia Warschburger and helpful discussions with Georg Jacob, Arne Wickenbrock, and Lykourgos Bougas. We further acknowledge support from the Deutsche Forschungsgemeinschaft (DFG) through the Deutsch-Israelische
Projektkooperation (DIP) program (Grant No. SCHM 1049/7-1) and the Bundesministerium für Bildung und Forschung via the program "IKT 2020 (Q.com)". This research is based upon work supported by the Office of the Director of National Intelligence (ODNI), Intelligence Advanced Research Projects Activity (IARPA), via the U.S. Army Research Office Grant No. W911NF-16-10070. The views and conclusions contained herein are those of the authors and should not be interpreted as necessarily representing the official policies or endorsements, either expressed or implied, of the ODNI, IARPA, or the U.S. Government.

\section{APPENDIX A: CONTRAST AND PHASE ESTIMATION}

As explained in Sec. II, the outcome of a measurement at a chosen interrogation time $T$ is described by the parity of the projected state. Assuming the state $\hat{\rho}$ [Eq. (2)], the probabilities to detect even parity for the $\hat{X}_{1} \hat{X}_{2}$ and $\hat{X}_{1} \hat{Y}_{2}$ measurements are given by

$$
\begin{aligned}
p_{X X}^{(E)} & =\operatorname{Tr}\left(\hat{R}_{Y, 1}\left(\frac{\pi}{2}\right) \hat{R}_{Y, 2}\left(\frac{\pi}{2}\right) \hat{\rho} \hat{R}_{Y, 1}^{\dagger}\left(\frac{\pi}{2}\right) \hat{R}_{Y, 2}^{\dagger}\left(\frac{\pi}{2}\right) \hat{P}_{E}\right) \\
& =\frac{1}{2}(1+C \cos (\varphi)), \\
p_{X Y}^{(E)} & =\operatorname{Tr}\left(\hat{R}_{Y, 1}\left(\frac{\pi}{2}\right) \hat{R}_{X, 2}\left(-\frac{\pi}{2}\right) \hat{\rho} \hat{R}_{Y, 1}^{\dagger}\left(\frac{\pi}{2}\right) \hat{R}_{X, 2}^{\dagger}\left(-\frac{\pi}{2}\right) \hat{P}_{E}\right) \\
& =\frac{1}{2}(1-C \sin (\varphi)),
\end{aligned}
$$

where $\hat{P}_{E}=|\uparrow \uparrow\rangle\langle\uparrow \uparrow|+| \downarrow \downarrow\rangle\langle\downarrow \downarrow|$ is the projector onto the subspace of even spin configurations, and $\hat{R}_{X / Y, i}(\theta)$ represent single qubit rotations by angle $\theta$ on ion $i=1,2$ prior to read-out. Equations (A1) can be inverted for $\phi$ and $C$; therefore, the measurement contains the maximum information about these parameters. However, as the measurements are subject to projection noise, statistical estimation of the phase is required. The read-out is dichotomic in terms of even or odd spin configurations, and the measurements are independent. Probing operators $\left\{\hat{X}_{1} \hat{X}_{2}, \hat{X}_{1} \hat{Y}_{2}\right\}$ each $\{N, M\}$ times, the probability to observe $\{n, m\}$ even spin configurations for given parameters $(\varphi, C)$ is given by binomial statistics:

$$
\begin{aligned}
& p_{X X}(n \mid \varphi, C)=\left(\begin{array}{c}
N \\
n
\end{array}\right) p_{X X}^{(E) n}\left(1-p_{X X}^{(E)}\right)^{N-n}, \\
& p_{X Y}(m \mid \varphi, C)=\left(\begin{array}{c}
M \\
m
\end{array}\right) p_{X Y}^{(E) m}\left(1-p_{X Y}^{(E)}\right)^{M-m} .
\end{aligned}
$$

For a measurement result $\{n, m\}$, the phase $\langle\varphi\rangle$ and contrast $\langle C\rangle$ are obtained by maximizing the likelihood function 


$$
L(\varphi, C)=L(n, m ; \varphi, C)=p_{X X}(n \mid \varphi, C) p_{X Y}(m \mid \varphi, C)
$$

with regards to $\varphi$ and $C$. If the sample sizes $N$ and $M$ are large, the likelihood ratio

$$
R(\varphi, C)=2 \log \left(\frac{L(\varphi, C)}{L(\langle\varphi\rangle,\langle C\rangle)}\right)
$$

is approximately $\chi^{2}$ distributed, such that $68.3 \%$ confidence intervals can be obtained via $R(\varphi,\langle C\rangle) \leq 1$ for $\varphi$ and $R(\langle\varphi\rangle, C) \leq 1$ for $C$.

\section{APPENDIX B: BAYESIAN EVALUATION}

In Bayesian statistics, the result after each phase measurement is described by the posterior PDF $\tilde{p}\left(\Delta \omega, \varphi_{0} \mid n, m ; T\right)$ [Eq. (4)]. The update function is given by

$$
p\left(n, m \mid \Delta \omega, \varphi_{0} ; T\right)=\int_{0}^{1} L\left(n, m ; \varphi\left(\Delta \omega, \varphi_{0} ; T\right), C\right) d C .
$$

For each parameter set $\left(\Delta \omega, \varphi_{0}\right)$, the accumulated phase after the interrogation time $T$ is given by

$$
\varphi\left(\Delta \omega, \varphi_{0} ; T\right)=\Delta \omega \times T+\varphi_{0} .
$$

Because of the phase periodicity, the update function features a $2 \pi / T$ periodicity in $\Delta \omega$. However, if the width of the prior PDF is smaller than the periodicity of the update function, the periodicity is not inherited by the posterior PDF. After at least two phase measurements at different interrogation times, the posterior PDF is well described by a two-dimensional normal distribution. To obtain estimates for $\Delta \omega$ and $\varphi_{0}$, we calculate expectation values from the marginalized PDF:

$$
\begin{aligned}
\langle\Delta \omega\rangle & =\iint \Delta \omega \cdot \tilde{p}\left(\Delta \omega, \varphi_{0} \mid n, m ; T\right) d \Delta \omega d \varphi_{0}, \\
\left\langle\varphi_{0}\right\rangle & =\iint \varphi_{0} \cdot \tilde{p}\left(\Delta \omega, \varphi_{0} \mid n, m ; T\right) d \Delta \omega d \varphi_{0} .
\end{aligned}
$$

Standard errors are obtained in a similar way by calculating the corresponding standard deviations.

\section{APPENDIX C: BAYESIAN EXPERIMENTAL DESIGN}

To calculate the optimal interrogation time $T$ for the next measurement to be performed, we employ Bayes' rule to calculate the posterior PDF for a hypothetical measurement result $\{n, m\}$ at interrogation time $T$ with fixed contrast $C$ :
$\tilde{p}\left(\Delta \omega, \varphi_{0} \mid n, m ; C, T\right)=\frac{p\left(n, m \mid \Delta \omega, \varphi_{0} ; C, T\right) p\left(\Delta \omega, \varphi_{0}\right)}{p(n, m \mid C, T)}$

with the marginal PDF

$$
\begin{aligned}
p(n, m \mid C, T)= & \iint p\left(n, m \mid \Delta \omega, \varphi_{0} ; C, T\right) \\
& \times p\left(\Delta \omega, \varphi_{0}\right) d \Delta \omega d \varphi_{0} .
\end{aligned}
$$

Here, it is sufficient to consider $N=M=1$ to save computational effort. Because we are interested in minimizing the error in $\Delta \omega$, we marginalize

$$
\tilde{p}(\Delta \omega):=\int \tilde{p}\left(\Delta \omega, \varphi_{0} \mid n, m ; C, T\right) d \varphi_{0} .
$$

Utility is defined as the expected gain in Shannon information of the posterior with respect to the prior after a hypothetical measurement,

$$
U(n, m ; T)=\int \tilde{p}(\Delta \omega) \log \tilde{p}(\Delta \omega) d \Delta \omega-U_{0},
$$

with the Shannon information of the marginalized prior PDF,

$$
U_{0}=\int p(\Delta \omega) \log p(\Delta \omega) d \Delta \omega
$$

Then, we average the utility function over all possible measurement results, weighted with the respective marginal probability:

$$
U(T)=\sum_{n=0}^{N} \sum_{m=0}^{M} w(T) U(n, m ; T) p(n, m \mid C, T) .
$$

Here, a penalty factor $w(T)=D(0) / D(T)$ takes the increased measurement duration for longer interrogation times into account, where $D(T)$ is the duration of a single experimental run with a given $T$. The ideal interrogation time for an upcoming measurement is $T_{0}=\max _{T} U(T)$; i.e., $T_{0}$ maximizes the expected gain in Shannon information. Via the known results from the prior PDF $\langle\Delta \omega\rangle$ and $\left\langle\varphi_{0}\right\rangle$, we add a phase offset to the second $X$ or $Y$ analysis pulse, such that the measured phase is always close to $\pi / 4$. Near $\pi / 4$, the error bar of a single phase measurement is minimized (at the expense of an increased contrast uncertainty).

[1] R. C. Jaklevic, J. Lambe, A. H. Silver, and J. E. Mercereau, Quantum Interference Effects in Josephson Tunneling, Phys. Rev. Lett. 12, 159 (1964). 
[2] D. Drung, High-Performance $d c$ Squid Read-Out Electronics, Physica (Amsterdam) 368C, 134 (2002).

[3] L. E. Fong, J. R. Holzer, K. K. McBride, E. A. Lima, F. Baudenbacher, and M. Radparvar, High-Resolution Room-Temperature Sample Scanning Superconducting Quantum Interference Device Microscope Configurable for Geological and Biomagnetic Applications, Rev. Sci. Instrum. 76, 053703 (2005).

[4] F. Baudenbacher, L. E. Fong, J. R. Holzer, and M. Radparvar, Monolithic Low-Transition-Temperature Superconducting Magnetometers for High Resolution Imaging Magnetic Fields of Room Temperature Samples, Appl. Phys. Lett. 82, 3487 (2003).

[5] M. E. Huber, N. C. Koshnick, H. Bluhm, L. J. Archuleta, T. Azua, P. G. Björnsson, B. W. Gardner, S. T. Halloran, E. A. Lucero, and K. A. Moler, Gradiometric MicroSQUID Susceptometer for Scanning Measurements of Mesoscopic Samples, Rev. Sci. Instrum. 79, 053704 (2008).

[6] D. Vasyukov, Y. Anahory, L. Embon, D. Halbertal, J. Cuppens, L. Neeman, A. Finkler, Y. Segev, Y. Myasoedov, M.L. Rappaport et al., A Scanning Superconducting Quantum Interference Device with Single Electron Spin Sensitivity, Nat. Nanotechnol. 8, 639 (2013).

[7] D. Budker and M. Romalis, Optical Magnetometry, Nat. Phys. 3, 227 (2007).

[8] W. Wasilewski, K. Jensen, H. Krauter, J. J. Renema, M. V. Balabas, and E.S. Polzik, Quantum Noise Limited and Entanglement-Assisted Magnetometry, Phys. Rev. Lett. 104, 133601 (2010).

[9] H. B. Dang, A. C. Maloof, and M. V. Romalis, Ultrahigh Sensitivity Magnetic Field and Magnetization Measurements with an Atomic Magnetometer, Appl. Phys. Lett. 97, 151110 (2010).

[10] I. K. Kominis, T. W. Kornack, J. C. Allred, and M. V. Romalis, A Subfemtotesla Multichannel Atomic Magnetometer, Nature (London) 422, 596 (2003).

[11] W. C. Griffith, S. Knappe, and J. Kitching, Femtotesla Atomic Magnetometry in a Microfabricated Vapor Cell, Opt. Express 18, 27167 (2010).

[12] A. Horsley, G. Du, and P. Treutlein, Widefield Microwave Imaging in Alkali Vapor Cells with Sub-100 $\mu \mathrm{m}$ Resolution, New J. Phys. 17, 112002 (2015).

[13] M. Koschorreck, M. Napolitano, B. Dubost, and M. W. Mitchell, High Resolution Magnetic Vector-Field Imaging with Cold Atomic Ensembles, Appl. Phys. Lett. 98, 074101 (2011).

[14] M. Vengalattore, J. M. Higbie, S. R. Leslie, J. Guzman, L. E. Sadler, and D. M. Stamper-Kurn, High-Resolution Magnetometry with a Spinor Bose-Einstein Condensate, Phys. Rev. Lett. 98, 200801 (2007).

[15] D. Simin, V. A. Soltamov, A. V. Poshakinskiy, A. N. Anisimov, R. A. Babunts, D. O. Tolmachev, E. N. Mokhov, M. Trupke, S. A. Tarasenko, A. Sperlich et al., All-Optical dc Nanotesla Magnetometry Using Silicon Vacancy Fine Structure in Isotopically Purified Silicon Carbide, Phys. Rev. X 6, 031014 (2016).

[16] T. Wolf, P. Neumann, K. Nakamura, H. Sumiya, T. Ohshima, J. Isoya, and J. Wrachtrup, Subpicotesla Diamond Magnetometry, Phys. Rev. X 5, 041001 (2015).
[17] V. M. Acosta, E. Bauch, A. Jarmola, L. J. Zipp, M. P. Ledbetter, and D. Budker, Broadband Magnetometry by Infrared-Absorption Detection of Nitrogen-Vacancy Ensembles in Diamond, Appl. Phys. Lett. 97, 174104 (2010).

[18] A. Angerer, T. Nöbauer, G. Wachter, M. Markham, A. Stacey, J. Majer, J. Schmiedmayer, and M. Trupke, Subnanotesla Quantum-Interference Magnetometry with a Single Spin in Diamond, arXiv:1509.01637.

[19] G. Balasubramanian, I. Y. Chan, R. Kolesov, M. Al-Hmoud, J. Tisler, C. Shin, C. Kim, A. Wojcik, P. R. Hemmer, A. Krueger, T. Hanke, A. Leitenstorfer, R. Bratschitsch, F. Jelezko, and J. Wrachtrup, Nanoscale Imaging Magnetometry with Diamond Spins under Ambient Conditions, Nature (London) 455, 648 (2008).

[20] M. S. Grinolds, S. Hong, P. Maletinsky, L. Luan, M. D. Lukin, R. L. Walsworth, and A. Yacoby, Nanoscale Magnetic Imaging of a Single Electron Spin under Ambient Conditions, Nat. Phys. 9, 215 (2013).

[21] M. Pelliccione, A. Jenkins, P. Ovartchaiyapong, Ch. Reetz, E. Emmanouilidou, N. Ni, and A.C. Bleszynski Jayich, Scanned Probe Imaging of Nanoscale Magnetism at Cryogenic Temperatures with a Single-Spin Quantum Sensor, Nat. Nanotechnol. 11, 700 (2016).

[22] U. Warring, C. Ospelkaus, Y. Colombe, K. R. Brown, J. M. Amini, M. Carsjens, D. Leibfried, and D. J. Wineland, Techniques for Microwave Near-Field Quantum Control of Trapped Ions, Phys. Rev. A 87, 013437 (2013).

[23] M. J. Biercuk, H. Uys, A. P. VanDevender, N. Shiga, W. M. Itano, and J. J. Bollinger, Optimized Dynamical Decoupling in a Model Quantum Memory, Nature (London) 458, 996 (2009).

[24] I. Baumgart, J.-M. Cai, A. Retzker, M. B. Plenio, and Ch. Wunderlich, Ultrasensitive Magnetometer Using a Single Atom, Phys. Rev. Lett. 116, 240801 (2016).

[25] S. Kotler, N. Akerman, Y. Glickman, A. Keselman, and R. Ozeri, Single-Ion Quantum Lock-In Amplifier, Nature (London) 473, 61 (2011).

[26] D. Sheng, A. R. Perry, S. P. Krzyzewski, S. Geller, J. Kitching, and S. Knappe, A Microfabricated OpticallyPumped Magnetic Gradiometer, Appl. Phys. Lett. 110, 031106 (2017).

[27] C. Granata, A. Vettoliere, C. Nappi, M. Lisitskiy, and M. Russo, Long Baseline Planar Superconducting Gradiometer for Biomagnetic Imaging, Appl. Phys. Lett. 95, 042502 (2009).

[28] S. M. Blakley, I. V. Fedotov, S. Ya. Kilin, and A. M. Zheltikov, Room-Temperature Magnetic Gradiometry with Fiber-Coupled Nitrogen-Vacancy Centers in Diamond, Opt. Lett. 40, 3727 (2015).

[29] C. F. Roos, M. Chwalla, K. Kim, M. Riebe, and R. Blatt, Designer Atoms for Quantum Metrology, Nature (London) 443, 316 (2006).

[30] T. Unden, P. Balasubramanian, D. Louzon, Y. Vinkler, M. B. Plenio, M. Markham, D. Twitchen, A. Stacey, I. Lovchinsky, A. O. Sushkov et al., Quantum Metrology Enhanced by Repetitive Quantum Error Correction, Phys. Rev. Lett. 116, 230502 (2016).

[31] S. F. Huelga, C. Macchiavello, T. Pellizzari, A. K. Ekert, M. B. Plenio, and J.I. Cirac, Improvement of Frequency 
Standards with Quantum Entanglement, Phys. Rev. Lett. 79, 3865 (1997).

[32] D. Leibfried, M. D. Barrett, T. Schaetz, J. Britton, J. Chiaverini, W. M. Itano, J. D. Jost, C. Langer, and D. J. Wineland, Toward Heisenberg-Limited Spectroscopy with Multiparticle Entangled States, Science 304, 1476 (2004).

[33] J. A. Jones, S. D. Karlen, J. Fitzsimons, A. Ardavan, S. C. Benjamin, G. A. D. Briggs, and J. J. L. Morton, Magnetic Field Sensing Beyond the Standard Quantum Limit Using 10-Spin NOON States, Science 324, 1166 (2009).

[34] T. Monz, P. Schindler, J. T. Barreiro, M. Chwalla, D. Nigg, W. A. Coish, M. Harlander, W. Hänsel, M. Hennrich, and R. Blatt, 14-Qubit Entanglement: Creation and Coherence, Phys. Rev. Lett. 106, 130506 (2011).

[35] C. F. Roos, G. P. T. Lancaster, M. Riebe, H. Häffner, W. Hänsel, S. Gulde, C. Becher, J. Eschner, F. Schmidt-Kaler, and R. Blatt, Bell States of Atoms with Ultralong Lifetimes and Their Tomographic State Analysis, Phys. Rev. Lett. 92, 220402 (2004).

[36] C. Langer, R. Ozeri, J. D. Jost, J. Chiaverini, B. DeMarco, A. Ben-Kish, R. B. Blakestad, J. Britton, D. B. Hume, W. M. Itano et al., Long-Lived Qubit Memory Using Atomic Ions, Phys. Rev. Lett. 95, 060502 (2005).

[37] S. Kotler, N. Akerman, N. Navon, Y. Glickman, and R. Ozeri, Measurement of the Magnetic Interaction Between Two Bound Electrons of Two Separate Ions, Nature (London) 510, 376 (2014).

[38] D. Kielpinski, V. Meyer, M. A. Rowe, C. A. Sackett, W. M. Itano, C. Monroe, and D. J. Wineland, A Decoherence-Free Quantum Memory Using Trapped Ions, Science 291, 1013 (2001).

[39] H. Häffner, F. Schmidt-Kaler, W. Hänsel, C. F. Roos, T. Körber, M. Chwalla, M. Riebe, J. Benhelm, U. D. Rapol, C. Becher, and R. Blatt, Robust Entanglement, Appl. Phys. B 81, 151 (2005).

[40] S. Schulz, U. Poschinger, F. Ziesel, and F. Schmidt-Kaler, Sideband Cooling and Coherent Dynamics in a Microchip Multi-Segmented Ion Trap, New J. Phys. 10, 045007 (2008),

[41] T. Ruster, C. T. Schmiegelow, H. Kaufmann, C. Warschburger, F. Schmidt-Kaler, and U. G. Poschinger, A Long-Lived Zeeman Trapped-Ion Qubit, Appl. Phys. B 122, 254 (2016).

[42] U. G. Poschinger, G. Huber, F. Ziesel, M. Deiß, M. Hettrich, S. A. Schulz, K. Singer, G. Poulsen, M. Drewsen, R. J. Hendricks, and F. Schmidt-Kaler, Coherent Manipulation of $a{ }^{40} \mathrm{Ca}^{+}$Spin Qubit in a Micro Ion Trap, J. Phys. B 42, 154013 (2009).

[43] D. Leibfried, B. DeMarco, V. Meyer, D. Lucas, M. Barrett, J. Britton, W. M. Itano, B. Jelenković, C. Langer, T. Rosenband et al., Experimental Demonstration of a Robust, High-Fidelity Geometric Two Ion-Qubit Phase Gate, Nature (London) 422, 412 (2003).

[44] See Supplemental Material at http://link.aps.org/ supplemental/10.1103/PhysRevX.7.031050 for a detailed error discussion.

[45] H. Kaufmann, T. Ruster, C. T. Schmiegelow, F. SchmidtKaler, and U. G. Poschinger, Dynamics and Control of Fast
Ion Crystal Splitting in Segmented Paul Traps, New J. Phys. 16, 073012 (2014).

[46] T. Ruster, C. Warschburger, H. Kaufmann, C. T. Schmiegelow, A. Walther, M. Hettrich, A. Pfister, V. Kaushal, F. Schmidt-Kaler, and U. G. Poschinger, Experimental Realization of Fast Ion Separation in Segmented Paul Traps, Phys. Rev. A 90, 033410 (2014).

[47] A. Walther, F. Ziesel, T. Ruster, S. T. Dawkins, K. Ott, M. Hettrich, K. Singer, F. Schmidt-Kaler, and U. Poschinger, Controlling Fast Transport of Cold Trapped Ions, Phys. Rev. Lett. 109, 080501 (2012).

[48] N. M. Nusran, M. Ummal Momeen, and M. V. Gurudev Dutt, High-Dynamic-Range Magnetometry with a Single Electronic Spin in Diamond, Nat. Nanotechnol. 7, 109 (2012).

[49] G. Waldherr, J. Beck, P. Neumann, R. S. Said, M. Nitsche, M. L. Markham, D. J. Twitchen, J. Twamley, F. Jelezko, and J. Wrachtrup, High-Dynamic-Range Magnetometry with a Single Nuclear Spin in Diamond, Nat. Nanotechnol. 7, 105 (2012).

[50] C. Bonato, M. S. Blok, H. T. Dinani, D. W. Berry, M. L. Markham, D. J. Twitchen, and R. Hanson, Optimized Quantum Sensing with a Single Electron Spin Using Real-Time Adaptive Measurements, Nat. Nanotechnol. 11, 247 (2016).

[51] K. Macieszczak, M. Fraas, and R. Demkowicz-Dobrzanski, Bayesian Quantum Frequency Estimation in Presence of Collective Dephasing, New J. Phys. 16, 113002 (2014).

[52] N. Wiebe and Ch. Granade, Efficient Bayesian Phase Estimation, Phys. Rev. Lett. 117, 010503 (2016).

[53] J. M. Taylor, P. Cappellaro, L. Childress, L. Jiang, D. Budker, P. R. Hemmer, A. Yacoby, R. Walsworth, and M. D. Lukin, High-Sensitivity Diamond Magnetometer with Nanoscale Resolution, Nat. Phys. 4, 810 (2008).

[54] D. Budker, D. F. Kimball, and D. P. DeMille, Atomic Physics: An Exploration through Problems and Solutions, 2 nd ed. (Oxford University Press, Oxford, 2008), pp. 98-100.

[55] R. Freeman, S. P. Kempsell, and M. H. Levitt, Radiofrequency Pulse Sequences which Compensate Their Own Imperfections, J. Magn. Reson. 38, 453 (1980).

[56] G. Tommaseo, T. Pfeil, G. Revalde, G. Werth, P. Indelicato, and J.P. Desclaux, The $g_{\mathrm{J}}$-Factor in the Ground State of $\mathrm{Ca}^{+}$, Eur. Phys. J. D 25, 113 (2003).

[57] M. Chwalla, J. Benhelm, K. Kim, G. Kirchmair, T. Monz, M. Riebe, P. Schindler, A. S. Villar, W. Hänsel, C. F. Roos et al., Absolute Frequency Measurement of the ${ }^{40} \mathrm{Ca}^{+} 4 s^{2} S_{1 / 2}-3 d^{2} D_{5 / 2}$ Clock Transition, Phys. Rev. Lett. 102, 023002 (2009).

[58] A. Kreuter, C. Becher, G. P. T. Lancaster, A. B. Mundt, C. Russo, H. Häffner, C. Roos, W. Hänsel, F. Schmidt-Kaler, R. Blatt, and M. S. Safronova, Experimental and Theoretical Study of the $3 d^{2}$ D-Level Lifetimes of ${ }^{40} \mathrm{Ca}^{+}$, Phys. Rev. A 71, 032504 (2005).

[59] All quantities with a " $\Delta$ " denote differences with respect to sensing positions $x_{1}$ and $x_{2}$; we consistently omit these arguments.

[60] M. Bal, C. Deng, J.-L. Orgiazzi, F. R. Ong, and A. Lupascu, Ultrasensitive Magnetic Field Detection Using a Single Artificial Atom, Nat. Commun. 3, 1324 (2012). 
[61] N. Herschbach, K. Pyka, J. Keller, and T. E. Mehlstäubler, Linear Paul Trap Design for an Optical Clock with Coulomb Crystals, Appl. Phys. B 107, 891 (2012).

[62] C. W. Chou, D. B. Hume, J. C. J. Koelemeij, D. J. Wineland, and T. Rosenband, Frequency Comparison of Two HighAccuracy $\mathrm{Al}^{+}$Optical Clocks, Phys. Rev. Lett. 104, 070802 (2010).

[63] W. M. Itano, J. C. Bergquist, A. Brusch, S. A. Diddams, T. M. Fortier, T. P. Heavner, L. Hollberg, D. B. Hume, S. R. Jefferts, L. Lorini et al., Optical Frequency Standards Based on Mercury and Aluminum Ions, Proc. SPIE 6673, 667303(2007).

[64] H. Kaufmann, T. Ruster, C. T. Schmiegelow, M. A. Luda, V. Kaushal, J. Schulz, D. von Lindenfels, F. Schmidt-Kaler, and U. G. Poschinger, Fast Ion Swapping for QuantumInformation Processing, Phys. Rev. A 95, 052319 (2017).

[65] F. Schmidt-Kaler and R. Gerritsma, Entangled States of Trapped Ions Allow Measuring the Magnetic Field Gradient Produced by a Single Atomic Spin, Europhys. Lett. 99, 53001 (2012).

[66] L. Thomas, F. Lionti, R. Ballou, D. Gatteschi, R. Sessoli, and B. Barbara, Macroscopic Quantum Tunnelling of Magnetization in a Single Crystal of Nanomagnets, Nature (London) 383, 145 (1996).

[67] M. Brownnutt, M. Kumph, P. Rabl, and R. Blatt, Ion-Trap Measurements of Electric-Field Noise Near Surfaces, Rev. Mod. Phys. 87, 1419 (2015). 